\title{
A modelling framework to project future climate change impacts on streamflow variability and extremes in the West River, China
}

\author{
YUAN FEI ${ }^{1,2}$, TUNG YEOU-KOUNG ${ }^{2}$ \& REN LILIANG ${ }^{1}$ \\ 1 State Key Laboratory of Hydrology-Water Resources and Hydraulic Engineering, College of Hydrology and Water \\ Resources, Hohai University, 1 Xikang Road, Nanjing, China \\ fyuan@hhu.edu.cn \\ 2 Department. of Civil and Environmental Engineering, The Hong Kong University of Science and Technology, Clear \\ Water Bay, Kowloon, Hong Kong SAR, China
}

\begin{abstract}
In this study, a hydrological modelling framework was introduced to assess the climate change impacts on future river flow in the West River basin, China, especially on streamflow variability and extremes. The modelling framework includes a delta-change method with the quantile-mapping technique to construct future climate forcings on the basis of observed meteorological data and the downscaled climate model outputs. This method is able to retain the signals of extreme weather events, as projected by climate models, in the constructed future forcing scenarios. Fed with the historical and future forcing data, a largescale hydrologic model (the Variable Infiltration Capacity model, VIC) was executed for streamflow simulations and projections at daily time scales. A bootstrapping resample approach was used as an indirect alternative to test the equality of means, standard deviations and the coefficients of variation for the baseline and future streamflow time series, and to assess the future changes in flood return levels. The West River basin case study confirms that the introduced modelling framework is an efficient effective tool to quantify streamflow variability and extremes in response to future climate change.
\end{abstract}

Key words climate change; streamflow; variability; flood; delta-change method; bootstrapping

\section{INTRODUCTION}

Many studies indicate that the risk of both floods and droughts is very likely to rise in many parts of the world under a warmer climate with increased climate variability (IPCC 2007). To effectively manage our water resources and adapt to climate change induced natural hazards, investigation of the global warming effects on future hydro-climate extreme events, the possible changes in future streamflow variability and extremes is essential. Such investigation is usually achieved by performing macro-scale hydrological modelling, driven by climate scenario datasets from either general circulation models (GCMs) or regional climate models (RCMs).

This paper presents a hydrological modelling framework to project the changes in future river flow induced by climate change, with an emphasis on the changes in streamflow variability and extreme flow events. The distinguishing features of this framework are: (1) a quantile-based deltachange method is included to construct future precipitation data that are able to preserve the original signals of precipitation changes projected by climate models; and (2) a bootstrapping approach is used to effectively test the significance of the changes in streamflow mean, standard deviation (SD) and the coefficients of variation (CV) and to estimate the uncertainties in future extreme flood projections.

\section{METHODS}

Figure 1 demonstrates the main procedures to quantify the watershed hydrologic variations in response to future climate change. First, a quantile-based delta-change method is applied to the baseline and future climate outputs from climate models (CMs) such as the Providing Regional Climates for Impacts Studies (PRECIS) regional climate model (RCM; Metoffice 2002) for analysing future climate-change signals and to construct the future precipitation and air temperature datasets. Subsequently, the observed historical and future climatic datasets are used to drive the Variable Infiltration Capacity (VIC) model (Liang et al. 1994) for simulating historical streamflows and future river flows. Finally, the projected future river flows are compared with the simulated historical streamflows to quantify the possible streamflow changes, in which the 


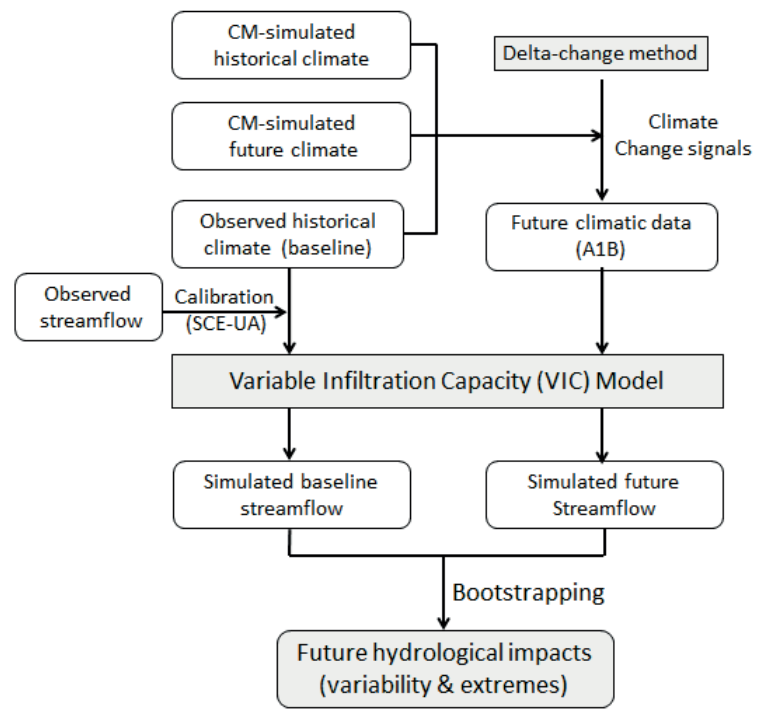

Fig. 1 Modelling framework for assessing possible future climate change impacts on streamflow.

bootstrap resampling method is used to test the significance of changes in future runoff means, SDs, CVs and extreme high flows. The descriptions of the quantile-based delta-change method, the VIC model and the bootstrapping technique are given as follows.

\section{Quantile-based delta-change method}

The delta approach was employed to construct a future climate dataset. First, the differences between CM-simulated baseline and future air temperature/precipitation are calculated as the projected future climate-change signals. Subsequently, the future climatic dataset for driving the VIC hydrological model is constructed by superimposing these signals on the observed historical climatic data. For instance, future daily air temperature as input to the VIC model is computed by applying the mean monthly anomalies between the CM-simulated baseline and future air temperature on the observed historical daily air temperature; future daily precipitation is calculated by multiplying observed precipitation with a quantile-based delta-change factor. The baseline climatology, used as the input of the hydrological model for baseline runs, corresponds to the observed meteorological dataset. The quantile-based delta-change method for constructing future daily precipitation time series is described in detail below.

The future daily precipitation time series are constructed by linearly scaling the observed historical daily precipitation with a quantile-based delta factor $\Delta_{p}$ at a daily time scale. For any one of the twelve months, the delta factor for scaling the observed historical precipitation quantile at each grid cell is defined as the ratio of the CM-simulated future daily precipitation to the CMsimulated baseline precipitation at the same quantile level. The constructed baseline and future daily precipitation time series are given by:

$$
\begin{aligned}
& P_{b s}(m, d)=P_{o b s}(m, d) \\
& P_{f u t}(m, d)=P_{o b s}(m, d) \times \Delta_{p} \\
& \Delta_{p}=P_{C M, f u t}\left(m, C D F_{o b s}\right) / P_{C M, b s}\left(m, C D F_{o b s}\right)
\end{aligned}
$$

where $P_{b s}(m, d)$ and $P_{f u t}(m, d)$ represent the constructed baseline and future daily precipitation on the $d$ th day of month $m$, respectively, and are used as the inputs to the hydrological model; $C D F_{\text {obs }}$ represents the cumulative probability of the observed historical precipitation on the $d$ th day of month $m\left(P_{o b s}(m, d)\right)$; and $P_{C M, b s}\left(m, C D F_{o b s}\right)$ and $P_{C M, f u t}\left(m, C D F_{o b s}\right)$ are, respectively, the CM-simulated daily precipitations for the baseline and future at the cumulative probability $C D F_{o b s}$. Equation (3) shows that the delta factor $\Delta_{p}$ is variable for the precipitation events at 
various levels, instead of using a constant factor for all precipitation events as adopted in traditional delta-change methods.

\section{The Variable Infiltration Capacity model}

The VIC model (Liang et al. 1994) is a state-of-the-art physically-based land surface model. It simulates a single vertical column of vegetation, snow and soil at each land grid cell. Radiative fluxes, turbulent fluxes of momentum, sensible heat and evapotranspiration are calculated at each time step. In the vertical soil column, physical processes such as heat diffusion, unsaturated liquid water transport, saturated gravitational drainage, local surface runoff, bottom drainage, uptake of liquid water by plant roots for transpiration, and freezing and thawing of soil ice, are calculated in the VIC model. The conceptual ARNO baseflow model (Todini 1996) is included in the VIC model to simulate baseflow from the deepest soil layer. A conceptual parameterization developed by Liang and Xie (2001) represents the surface runoff generation by effectively combining the infiltration excess runoff mechanism with the saturation excess runoff mechanism.

\section{Bootstrapping for confidence intervals of runoff-change statistics}

To quantify future streamflow change, three runoff-change statistics are defined at annual and monthly time scales, in terms of runoff mean, standard deviation (SD) and the coefficient of variation (CV). They are: (1) $f_{\text {MEAN }}$, the ratio of the projected future runoff mean $\left(\bar{R}_{f u t}\right.$, in mm) to the baseline runoff mean $\left(\bar{R}_{B S}\right.$, in $\left.\mathrm{mm}\right)$; (2) $f_{S D}$, the ratio of the projected future runoff SD $\left(S D_{f u t}\right.$ in $\left.\mathrm{mm}\right)$ to the simulated baseline runoff $\mathrm{SD}\left(S D_{B S}\right.$, in $\left.\mathrm{mm}\right)$; and (3) $f_{C V}$, the ratio of the projected future runoff $\mathrm{CV}\left(C V_{f u t}\right)$ to the simulated baseline runoff $\mathrm{CV}\left(C V_{B S}\right)$.

Bootstrapping is a distribution-free resampling method for assessing the accuracy to sample estimates (such as confidence intervals for the sample mean and standard deviation) by randomly sampling with replacement from observed samples or time series (Efron 1979). To estimate the confidence interval (CI) for a runoff-change statistic, the centred bootstrap percentile method (Lunneborg 2000) is used; it is applicable to the situation when the sampling distribution of a statistic obtained by bootstrapping might be highly skewed. The procedures are as follows:

(1) Calculate the original sample value of a runoff-change statistic, denoted as $\hat{f}$, from the VICsimulated annual/monthly runoff sample pair $(N$ years both for baseline runoff and future runoff).

(2) Generate pair-wise bootstrap runoff samples by randomly drawing with replacement for both the baseline and future runoff (taking corresponding years from the $N$-year simulations).

(3) Compute the estimate of the runoff-change statistic for the bootstrap runoff sample pair.

(4) Repeat steps (2) and (3) for $M$ times (20 000 times in the case study) to produce $M$ bootstrap estimates for that statistic.

(5) Arrange the $M$ bootstrap statistic estimates in ascending order to find the $\alpha / 2$ and $1-\alpha / 2$ quantiles $\left(f_{\alpha}^{b o o t}\right.$ and $\left.f_{1-\alpha}^{b o o t}\right)$, and calculate the lower and upper confidence limits of the runoff change statistic $\left(C I_{\text {lower }}\right.$ and $\left.C I_{\text {upper }}\right)$ as:

$$
\begin{aligned}
& C I_{\text {lower }}=2 \hat{f}-f_{1-\alpha}^{\text {boot }} \\
& C I_{\text {upper }}=2 \hat{f}-f_{\alpha}^{\text {boot }}
\end{aligned}
$$

The estimated CIs of these runoff change statistics are analysed to determine whether the projected future runoff mean, SD and CV are significantly different from the corresponding baseline runoff statistics. Here, the CI of $f_{M E A N}$ is taken as an example. If the $1-\alpha$ confidence interval of $f_{M E A N}$ includes 1.0, it indicates that the projected mean runoff under a future scenario does not differ significantly from the baseline mean at a $\alpha$ significance level. If the lower confidence limit of $f_{M E A N}$ is $>1.0$, then the future mean runoff can be considered significantly larger than the baseline mean. Similarly, if the upper confidence limit of $f_{M E A N}$ is below 1.0, it implies that the future mean runoff is expected to be significantly lower than the baseline mean runoff. 


\section{Bootstrapping for uncertainties in extreme flood projections}

The bootstrapping method is used to quantify the uncertainty in estimating the changes in extreme flooding events. At first, paired bootstrap samples are generated, by randomly drawing with replacement from the original annual maximum daily streamflow samples under the baseline and future climates. Subsequently, the change of $T$-year flow is estimated by fitting a Pearson type III distribution to the resampled annual maximum flows for the baseline and future climates, and taking the difference between these two estimates. The whole procedure is repeated a large enough number of times (20 000 times in the case study) to derive a frequency distribution of possible predicted changes in the $T$-year flood event.

\section{STUDY AREA AND DATA PREPARATION}

The West River basin located in South China and having a total drainage area of $3.05 \times 10^{5} \mathrm{~km}^{2}$ was chosen as the case study area (Fig. 2). It has a tropical and subtropical climate. Historical meteorological data of 33 weather stations in the basin (Fig. 2) were obtained from the China Meteorological Administration, and include daily records of maximum and minimum air temperature and precipitation for 1961-1990. Observed daily streamflow data of 16 hydrologic stations (Fig. 2) during 1961-1989 were provided by the Ministry of Water Resources, China. The PRECIS RCM climate data were employed for deriving future climate change signals; they are composed of simulated daily maximum and minimum air temperature and precipitation in the baseline years (1961-1990) and future years (2011-2040) under the A1B scenario of the Intergovernmental Panel on Climate Change (IPCC) Special Report on Emission Scenarios (SRES) at a $50-\mathrm{km}$ spatial resolution. Linear interpolation was performed to transform the weather station data and PRECIS-simulated forcing data to a $0.25^{\circ}$ resolution.

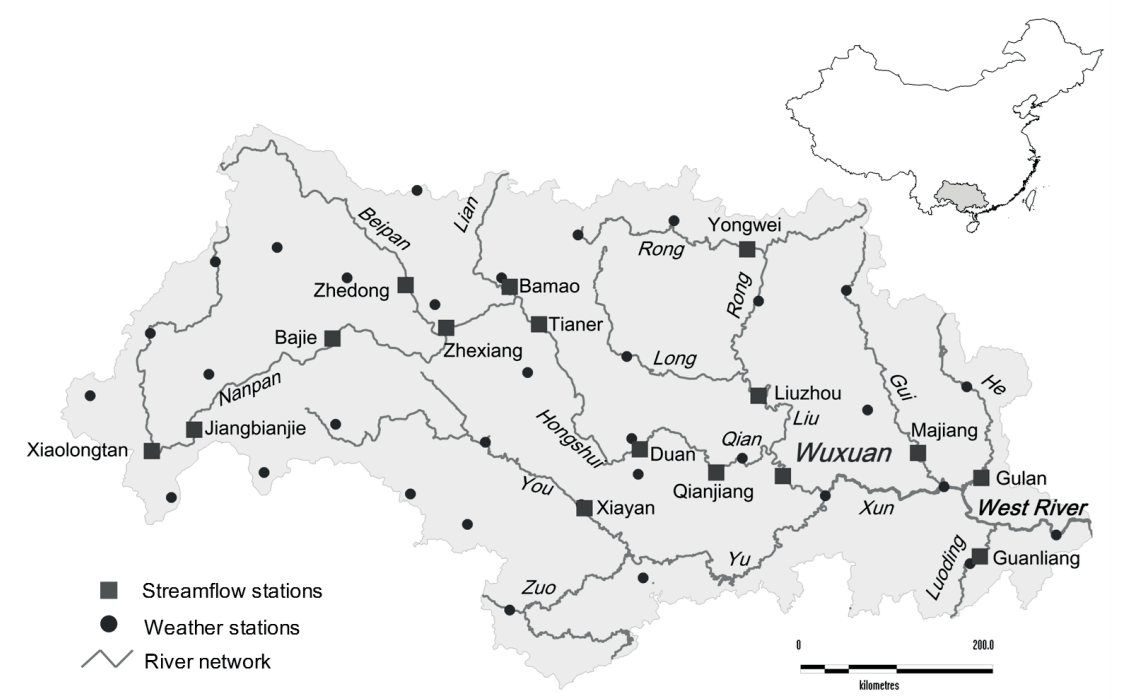

Fig. 2 Meteorological and streamflow stations in the West River basin.

\section{CASE STUDY RESULTS}

\section{Future air temperature and precipitation changes}

Relative to the baseline period (1961-1990), air temperature under the A1B scenario (2011-2040) undergoes a moderate increase of $0.9-1.5^{\circ} \mathrm{C}$ throughout the year (Fig. 3). The highest temperature increase appears in August and the lowest increase in October. Mean annual precipitation under the A1B scenario is projected to increase by $7.4 \%$ over the baseline situation. A considerable increase in precipitation would occur in most of the rainy season (May-September), whereas part of the dry season (November-February) is likely to become much drier, with the largest precipitation reduction, 29.1\%, in December. 


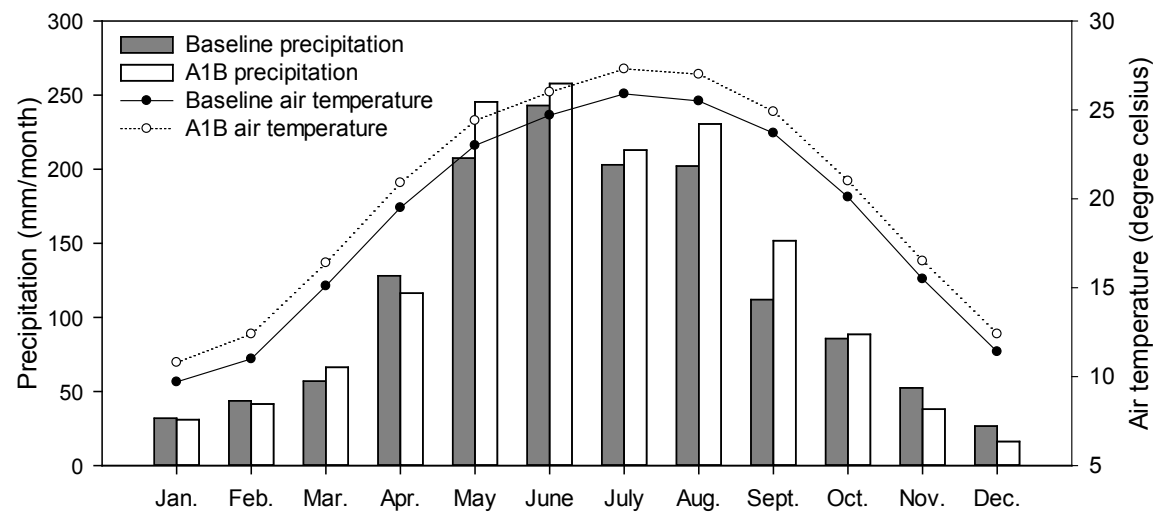

Fig. 3 Monthly mean air temperature and precipitation in the West River basin under baseline (1961 to 1990) and A1B (2011 to 2040) scenarios.

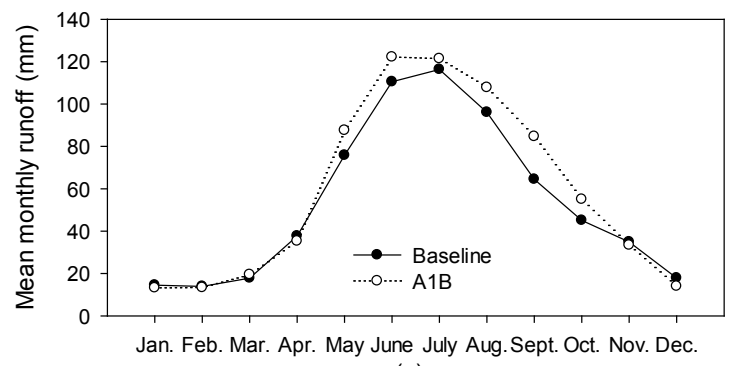

(a)

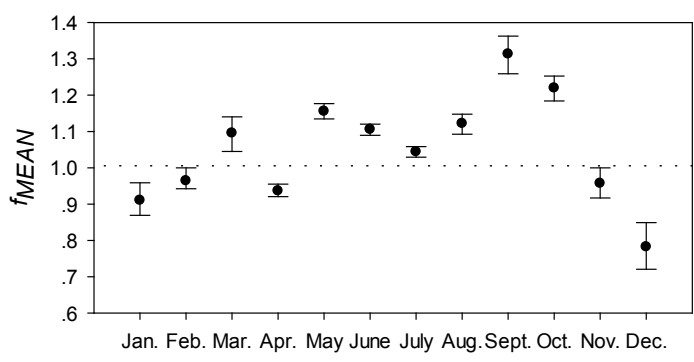

(b)

Fig. 4 Changes in mean monthly runoff in the Wuxuan watershed: (a) comparison between the baseline and A1B mean monthly runoffs; and (b) monthly runoff-change statistic $f_{M E A N}$ with its $95 \%$ CI.

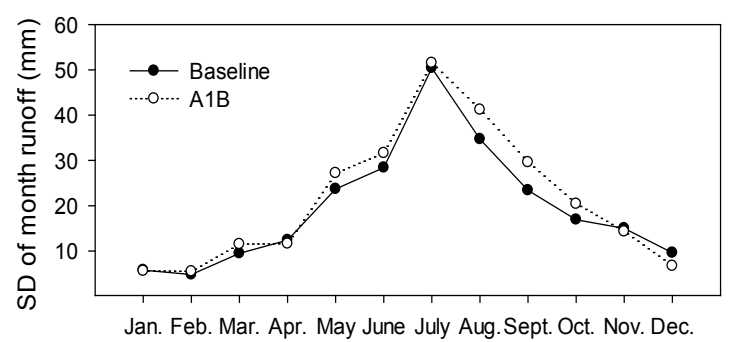

(a)

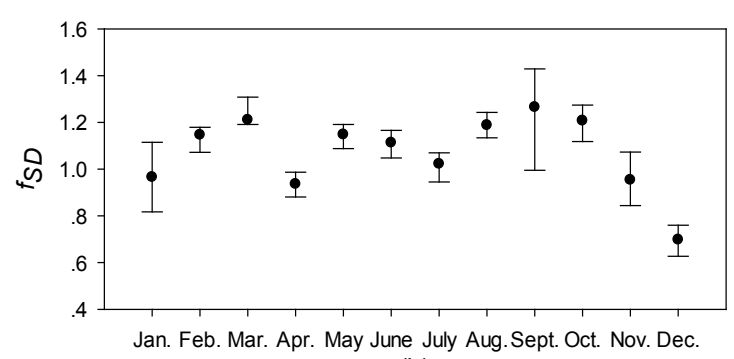

(b)

Fig. 5 Changes in monthly runoff SD in the Wuxuan watershed: (a) comparison between the baseline and A1B monthly runoff SDs; and (b) monthly runoff-change statistic $f_{S D}$ with its $95 \% \mathrm{CI}$.

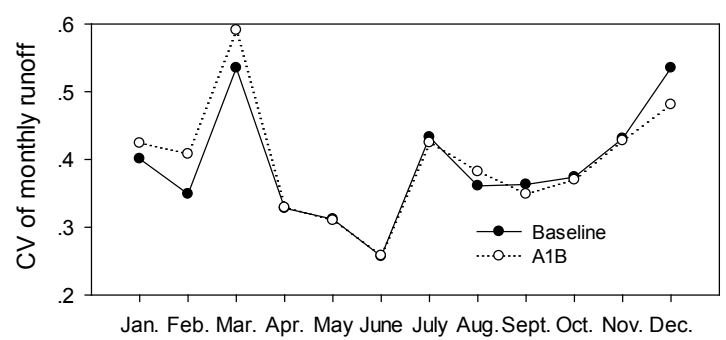

(a)

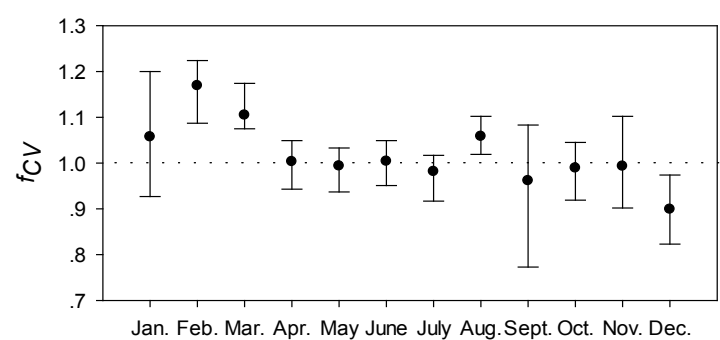

(b)

Fig. 6 Changes in monthly runoff $\mathrm{CV}$ in the Wuxuan watershed: (a) comparison between the baseline and A1B monthly runoff CVs; and (b) monthly runoff-change statistic $f_{C V}$ with its $95 \% \mathrm{CI}$.

\section{Future changes in streamflow}

Means of streamflow Figure 4 compares the calculated A1B monthly mean runoff at Wuxuan hydrologic station (Fig. 2) with the baseline one and shows the $95 \%$ CI of $f_{\text {MEAN }}$ for each 
month calculated by the 20000 bootstrapping resamples. It indicates that there is a significant increase in mean runoff in most months of the wet season (May-September) under the A1B scenario. At the beginning and end of the dry season (October and March, respectively), mean runoff in the future tends to rise significantly. Other months of the wet season are projected to have an increasing trend in mean runoff, with a significant negative change in December and January.

Streamflow variability With respect to the monthly runoff variability, a significant increase in runoff SDs is found in six months (February-March, May-June, August and October), and the April and December runoff SDs under A1B are significantly lower than those in baseline (Fig. 5). In terms of runoff $\mathrm{CV}$, a significant increase in runoff variability is only projected in February, March and August, while December would undergo a decrease in runoff variability (Fig. 6).

Streamflow extremes To predict the climate change impact on flood return levels, the simulated annual maximum daily streamflow series under the baseline and A1B scenarios were fitted by the Pearson type III distribution (Fig. 7). It demonstrates clear changes in the magnitude and frequency of extreme flooding events at Wuxuan station. The 100-year daily river flow under the A1B scenario increases by $10.9 \%$ over the baseline high at the same return period; the baseline 100 year event would be approximately a 50-year event in A1B. The extreme high flows at other return periods $(5,10,20$ and 50 years) in $\mathrm{A} 1 \mathrm{~B}$ are estimated to rise by $11.2-12.6 \%$ over the baseline extremes.

In this study, the bootstrapping technique with 20000 repetitions was used to test whether 30year streamflow simulations under both baseline and A1B scenarios are sufficient to project the future changes of flooding river flows at various return periods. With bootstrapping, the 100 -year maximum daily discharge under A1B would increase by $5640 \mathrm{~m}^{3} / \mathrm{s}$ on average (Fig. 7(b)), fairly consistent with the Pearson type III plots in Fig. 7(a) which indicate an increase of $5600 \mathrm{~m}^{3} / \mathrm{s}$. The bootstrapping method also gives a mean increase in the future extreme high flow at other return periods $(5,10,20$ and 50 years), with a similar magnitude of increase as shown in Fig. 7(b) Though an overall increase in extreme high river flow is estimated by bootstrapping, there is a considerable probability of predicting a decrease, particularly in the case of flood events at higher return periods. For the 100-year and 50-year discharges, this probability is, respectively, $9.5 \%$ and $6.6 \%$, whereas it decreases to $2.4 \%$ and $0.45 \%$ for the 20 -year and 10 -year events and the likelihood is just $0.02 \%$ for 5 -year flow. Figure 7(b) illustrates that the 100 -year and 50 -year events have a wider band of variation, while the 5 -year event has the narrowest band. This implies that the 30-year streamflow simulations for the baseline and A1B scenarios are too short and may not sufficiently capture the changes in flood events at higher return periods and larger sample sizes of annual maximum flow time series are required to reduce this uncertainty.

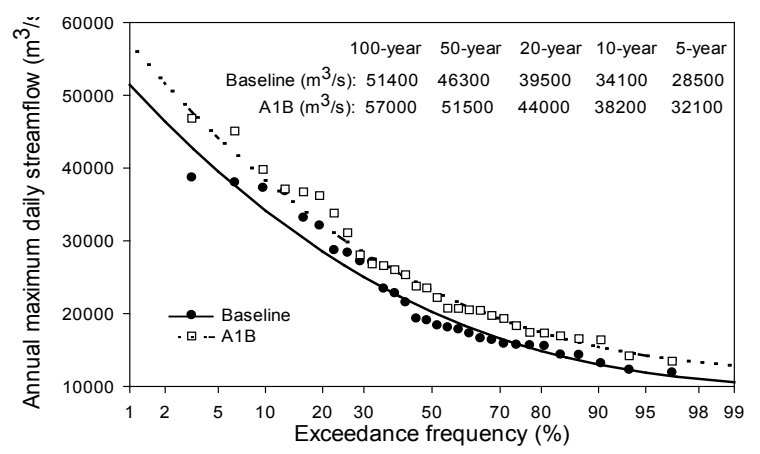

(a)

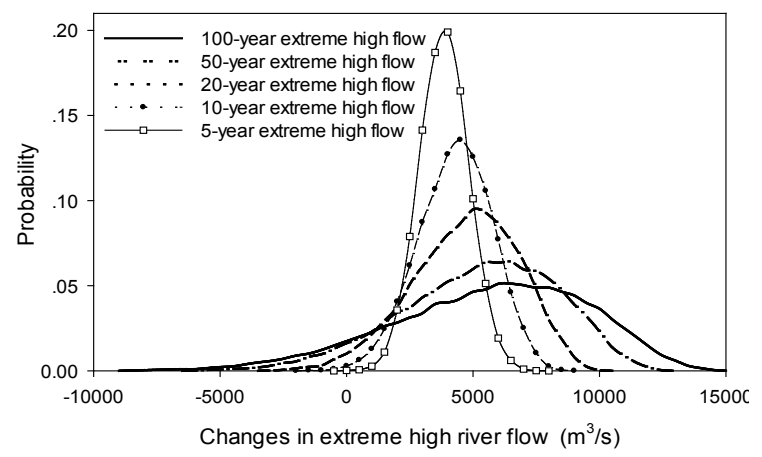

(b)

Fig. 7 Pearson type III plots of annual maximum daily discharge under baseline and A1B scenarios (a) and frequency distribution of possible predicted changes in extreme high flow at various return periods (b). 


\section{CONCLUSIONS}

A modelling framework was introduced to investigate climate change impacts on river flow, in particular on streamflow variability and extremes. A delta-change method with quantile-mapping was used to construct future forcing data, which is able to preserve the signals of the extreme weather events in future scenarios. Driven by the historical and future forcing data, the VIC model was used to simulate baseline streamflow and to project future streamflow at daily time scales. A bootstrapping approach was used to test whether the projected future streamflow differs significantly from the baseline river flow at monthly time scales, in terms of runoff mean, SD and $\mathrm{CV}$. This method was also applied to quantify the uncertainties of the predicted changes in extreme flood events that are caused by the limited size of annual extreme flow samples. The case study shows that the 30-year streamflow simulations for baseline and A1B scenarios leads to larger bands of variation of the extreme high flow changes at longer return periods. Therefore, hydrological simulations for longer periods are necessary so as to cut down this uncertainty.

Acknowledgements This study is sponsored by the Research Project Competition Grant (RPC10EG25) of the Hong Kong University of Science, the National Natural Science Foundation of China (Grant No. 50909033) and Technology and Special Fund of State Key Laboratory of Hydrology-Water Resources and Hydraulic Engineering (no. 2011585412).

\section{REFERENCES}

Efron, B. (1979) Bootstrap methods: Another look at the jackknife. Ann. Stat., 7, 1-26.

IPCC (2007) Climate Change 2007: The Physical Science Basis. Contribution of Working Group I to the Fourth Assessment Report of the Intergovernmental Panel on Climate Change. Cambridge University Press: Cambridge, UK.

Liang, X., et al. (1994)A simple hydrologically based model of land surface water and energy fluxes for general circulation models. Journal of Geophysical Research 99 (D7), 14 415-14 428.

Liang, X. \& Xie, Z. (2001) A new surface runoff parameterization with subgrid-scale soil heterogeneity for land surface models. Advances in Water Resources, 24, 1173-1193.

Lunneborg, C. E. (2000) Data analysis by resampling: concepts and applications. Duxbury Press: Pacific Grove, CA, USA.

Metoffice (2002) PRECIS-Update. Metoffice: London.

Todini, E. (1996). The ARNO rain-runoff model. Journal of Hydrology. 175, 339-382. 